\title{
Impatto da odori di impianti industriali: metodi analitici e sensoriali a confronto
}

\author{
Giusy Addeo ${ }^{1}$ \\ ${ }^{1}$ Affiliation not available
}

\begin{abstract}
La misura delle emissioni da odori e delle concentrazioni di sostanze odorigene si inserisce in uno scenario in cui i cattivi odori sono tra le principali fonti di disturbo per la popolazione esposta. Tali scenari vengono associati a condizioni di pericolo per la salute e per la qualità dell'aria. L'impatto da odori rappresenta una criticità degli impianti industriali come quelli di trattamento delle acque reflue, specialmente quando sono localizzati in zone fortemente urbanizzate, in tali circostanze risulta opportuna l'analisi dell'impatto odorigeno. La stima degli odori avviene con metodi analitici e sensoriali. Si propone la correlazione tra i suddetti metodi integrando la soggettività sensoriale con l'oggettività analitica. Nel presente articolo si mostrano varie applicazioni della comparazione dei metodi, adoperando diverse tecniche uliti per gestire e manutenere un impianto e per individuare nuove sostanze responsabili di odori sgradevoli.
\end{abstract}

\section{Introduzione ai metodi di misura degli odori}

Gli impianti di trattamento delle acque reflue sono sorgenti di cattivi odori che arrecano disturbo alla popolazione e, in alcuni casi, possono influenzare negativamente la qualità della vita. Diversi studi hanno dimostrato come le emissioni odorigene emanate da tali impianti,in certe circostanze, possano essere causa di stress, insonnia, perdita dell'appetito e comportamenti irrazionali. Anche se la maggior parte di questi odori non comporta rischi tossicologici e sanitari, perché in rari casi risultano pericolosi e perché le loro concentrazioni sono discrete, la popolazione li associa comunque a condizioni di area non salubri ${ }^{1}$.

Tale situazione è da considerarsi soprattutto quando non è possibile situare gli impianti ad un'opportuna distanza dai centri abitati (Fig.1), per cui bi- 
sogna affrontare nel dettaglio il problema della sostenibilità igienica relativa all'ubicazione dell'impianto ${ }^{2}$.

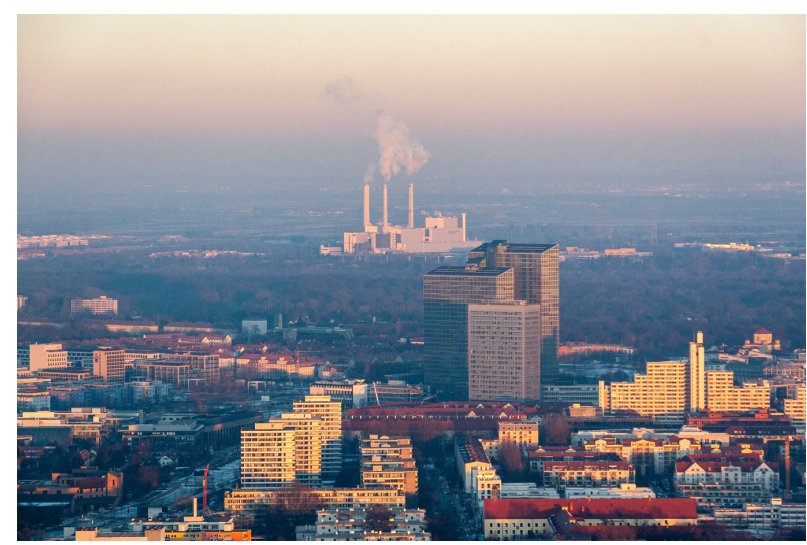

Figure 1: Emissioni da odori derivanti da impianti industriali non molto distanti dai centri urbani

In letteratura, per la misura degli odori, si distinguono metodi sensoriali (es. olfattometria dinamica e/o questionari sociologici) e analitico-strumentali (es. GC-MS, metodi colorimetrici). Con tecniche sensoriali non si ottengono risultati oggettivi, in quanto la sensibilità olfattiva è variabile da soggetto a soggetto, mentre con tecniche analitiche GC-MS si ottengono informazioni sui principali composti che costituiscono la miscela odorosa. Quindi è opportuno combinare la soggettività sensoriale con la precisione strumentale ${ }^{1}$.

In lavori bibliografici più recenti si presenta un'analisi quali-quantitativa dei campioni raccolti in fasi differenti di un impianto di trattamento delle acque reflue mediante il naso elettronico. II naso elettronico (E-Nose) è dotato di sensori che riconoscono odori semplici e complessi e, rispetto agli altri sistemi, consente l'analisi contemporanea di sostanze gassose e odori, con la possibilità di monitorare continuamente $i$ dati in ingresso e di restituire analisi qualiquantitative in uscita. Si mette in evidenza anche come l'utilizzo di tecniche GC-MS non consenta l'interazione delle diverse miscele gassose che producono gli odori e, pur essendo un metodo accurato, gli elevati costi e i limiti nell' esecuzione delle misure in laboratorio rappresentano degli elementi di debolezza che ne pregiudicano l'utilizzo. L' olfattometria, invece, risulta maggiormente impiegata, pur essendo dispendiosa per tempi e costi e meno accurata a causa della sua soggettività. L'analisi degli odori ambientali può essere supportata anche dalla combinazione di GC-MS e olfattometria o dalla combinazione di naso elettronico e olfattometria, tali combinazioni sono utili per la stima degli odori 
avvertiti dalle popolazioni ${ }^{3}$. Ulteriori studi mostrano come il modo migliore per caratterizzare gli odori, stimarne la concentrazione e sviluppare sistemi di monitoraggio innovativi sia dato dalla sinergia tra caratterizzazione chimica, olfattometria dinamica e i nasi elettronici ${ }^{4}$. Oggi, in ambito internazionale, esistono leggi che indicano $i$ limiti di emissioni odorigene derivanti da attività industriali. A livello europeo l'introduzione della norma EN 13725:2003 "Qualità dell'aria-misure della concentrazione da odori con I' olfattometria dinamica", ha individuato l' olfattometria dinamica come metodo per la determinazione oggettiva della concentrazione di odori in campioni gassosi percepiti da valutatori umani, con lo scopo di fornire una valutazione delle emissioni di odori comune in tutti i paesi dell'Unione Europea. La principale problematica relativa alla definizione di limiti normativi è connessa alla soggettività dell'olfatto ${ }^{5}$.

L'obiettivo dello studio analizzato è indagare sulla correlazione di analisi sensoriali ed analitiche per rilevare gli odori in modo quali-quantitativo e caratterizzarli obiettivamente.

\section{Tecniche sensoriali, strumentali e la loro combina- zione in diverse applicazioni}

Per effettuare un confronto tra metodi analitici e sensoriali sono state considerate le analisi condotte su campioni prelevati in un mese nell'impianto di trattamento delle acque reflue sito in un campus in Germania. I campioni sono stati prelevati con tre sacche di campionamento da 3 litri e sono stati posizionati in un contenitore rigido. Successivamente i campioni sono stati trasferiti in una rete polimerica porosa che assorbe i composti organici che poi vengono desorbiti termicamente da un gascromatografo, spurgando l'adsorbente e raccogliendo i composti in una trappola di raffreddamento. L'analisi sensoriale è stata svolta con il metodo dell' olfattometria dinamica mentre l'analisi strumentale è stata svolta con modello GC-MS. I risultati del metodo sensoriale hanno mostrato che l'impatto da odori è imputabile prevalentemente alla gestione dei fanghi, mentre i risultati del metodo analitico hanno evidenziato la presenza di circa 36 sostanze diverse all' interno della miscela volatile del GC-MS, di queste la metà sono state reputate le principali responsabili dell'odore tipico di un impianto di trattamento di acque reflue ${ }^{1}$.

Il disolfuro di dimetile risultava la sostanza volatile con la soglia d'odore più bassa e più rilevante nell' impianto, pertanto è stato definito composto "chiave", e la sua concentrazione più alta è stata rilevata nel fango ispessito.

Per misurare le emissioni da odori in modo analitico è stato proposto l' Indice 
Analitico degli Odori AOly :

$$
A O I y=\sum_{y=1}^{n} \frac{C y}{O T y}
$$

Quest'indice è funzione della concentrazione della sostanza volatile rilevata con metodo GC-MS (Cy) e della soglia d'odore (OTy).

Similmente è stato presentato I'Indice Sensoriale degli Odori SOly:

$$
S O I y=\frac{U i}{U s}
$$

Quest'indice è funzione della concentrazione di emissioni odorigene, all' i-esimo punto di campionamento, rilavate con olfattometro (Ui) e della concentrazione standard di emissioni odorigene rilevate con analisi sensoriali (Us).

Per tutte le analisi effettuate per ogni punto del campione rappresentando AOI in funzione di SOl è stato evidenziato un nesso tra i due indici ${ }^{1}$.

Analogamente sottoponendo i campioni a tecniche come olfattometria dinamica e naso elettronico, i rispettivi indici sono risultati confrontabili, a testimonianza del fatto che la caratterizzazione degli odori eseguita con i due metodi è comparabile. Per cui è possibile adottare il naso elettronico col vantaggio di effettuare un monitoraggio degli odori continuo ${ }^{6}$.

Tecniche sensoriali-strumentali combinate come gascromatografiaspettrometria di massa-olfattometrica (GC/MS-O) e gascromatografia multidimensionale-spettrometria di massa- olfattometrica (MDGC / MS-O), sono idonee all' identificazione di composti odorosi e la loro sinergia è efficace per la corretta gestione dei cattivi odori indotti da impianti industriali ${ }^{4}$.

Per analizzare la qualità dell'aria relativamente all' intensità degli odori, oltre a quelle già citate, sono note anche tecniche analitiche come gascromatografia con rilevazione olfattometrica (GC-O), che consente analisi qualitative e quantitative in parallelo, e tecniche sensoriali come l'olfattometro da campo (FO) che permette un'analisi istantanea delle sostanze odorose e delle emissioni da odori in sito. Una pecca di tali tecniche è che richiedono un team di esperti valutatori la cui sensibilità olfattiva può essere limitata, ciò nonostante sono tra le tecniche più diffuse per la stima degli odori emessi da diversi impianti ingegneristici ${ }^{7}$.

Un esempio dell' impiego di diverse tecniche di misura degli odori lo si ritrova in Polonia, dove per la stima dell'impatto da odori in 3 impianti di trattamento 


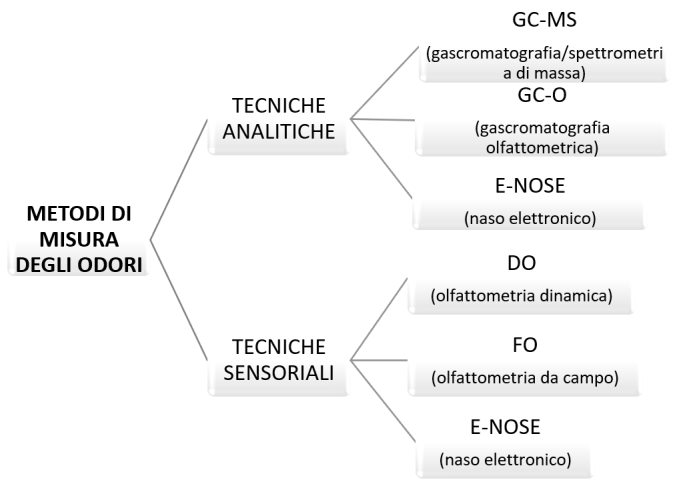

Figure 2: Tecniche di valutazione dell'intensità degli odori (schema adattato dalla Fig. $3 \mathrm{di}^{7}$ )

di acque reflue, sono stati messi a confronto 2 analisi sensoriali (olfattometria di campo FO e olfattometria dinamica DO), e 2 analisi chimiche (determinazione colorimetrica dell'idrogeno solforato e ammoniaca, e determinazione dei composti specifici dello zolfo mediante GC-MS) ${ }^{8}$.

In futuro l'impiego di tecniche come il naso elettronico potrebbe rivelarsi uno strumento opportuno all' individuazione di anomalie in un impianto, consentendo interventi tempestivi e minimizzando gli odori sgradevoli. Questo sarebbe d'ausilio alla gestione degli odori e alla programmazione delle attività di manutenzione 37 .

Tecniche analitiche e sensoriali sono state applicate anche in campi diversi da quello delle emissioni odorigene generate da impianti industriali, un esempio è uno studio condotto su campioni di legno del pino silvestre ${ }^{9}$, che ha identificato i principali composti odorosi responsabili dell'odore caratteristico del legno. L'impatto da odore stimato è dovuto in gran parte ai terpeni come l' $\alpha$-pinene che è il principale composto organico presente nelle resine di piante conifere responsabile dell'odore legnoso. Nello studio sono state scoperte 11 nuove sostanze responsabili del odore del legno a cui si associa un odore agrumato (linalolo), grassoso (dienale), ammuffito (borneolo), di formaggio (acido pentanoico), erboso (pentanale), fruttato-metallico (timolo), simile al cocco ( $\delta$-nonalactone e $\gamma$-octalactone $)$, simile alle pesche $(\gamma$-decalactone e dodecalactone), di sapone (acido dodecanoico). 


\section{Conclusioni}

Per valutare nel dettaglio gli odori percepiti dalla popolazione e la caratterizzazione chimica dei composti odorosi è opportuno un approccio sinergico tra tecniche analitiche e sensoriali, tale approccio si dimostra vincente per individuare gli odori emessi da impianti industriali e realizzare sistemi di monitoraggio attendibili ${ }^{4}$.

Il confronto tra tecniche GC-MS e sensoriali delle sostanze volatili, che si originano in un impianto di trattamento delle acque reflue, viene affrontato tramite la definizione di indici che dimostrano la comparabilità dei metodi ${ }^{6}$

Allo stesso tempo l'utilizzo di metodi differenti è causa di interpretazioni incongruenti dei risultati soprattutto in mancanza di riferimenti normativi in merito ${ }^{8}$. In tal senso in diversi paesi sono stati adottati degli standard che tengono conto di norme sulle emissioni, della distanza minima tra centri abitati e fonti di emissioni, e dei reclami da parte della popolazione esposta ${ }^{7}$.

Si prospetta che in futuro la ricerca scientifica possa orientarsi verso metodi innovativi volti ad eliminare la componente soggettiva nella misura degli odori ${ }^{3}$.

\section{References}

1.T.Zarra, V.Naddeo, V.Belgiorno, M.Reiser \& M.Kranert. Instrumental characterization of odour: a combination of olfactory and analytical methods. Water Science and Technology 59, 1603-1609 (2009).

2.P.Stellacci, L.Liberti, M.Notarnicola \& C.N.Haas. Hygienic sustainability of site location of wastewater treatment plants. Desalination 253, 51-56 (2010).

3.A.Blanco-Rodríguez et al.. Development of an electronic nose to characterize odours emitted from different stages in a wastewater treatment plant. Water Research 134, 92-100 (2018).

4.P.Giungato et al.. Synergistic approaches for odor active compounds monitoring and identification: State of the art integration, limits and potentialities of analytical and sensorial techniques. TrAC Trends in Analytical Chemistry 107, 116-129 (2018).

5.T.Zarra, V.Naddeo, V.Belgiorno, M.Reiser \& M.Kranert. Odour monitoring of small wastewater treatment plant located in sensitive environment. Water Science and Technology 58, 89-94 (2008). 
6.T.Zarra, V.Naddeo, M.Reiser \& M.Kranert. Odour emissions characterization from wastewater treatment plants by different measurement methods. Chemical Engineering Transactions 40,37-42, (2014).

7.Gebicki, J., Byliński, H. \& Namieśnik, J. Measurement techniques for assessing the olfactory impact of municipal sewage treatment plants. Environmental Monitoring and Assessment 188, (2015).

8.R.J.Barczak \& A.Kulig. Comparison of different measurement methods of odour and odorants used in the odour impact assessment of wastewater treatment plants in Poland. Water Science and Technology 75, 944-951 (2016).

9.L.Schreiner, P.Bauer \& A.Buettner. Resolving the smell of wood - identification of odour-active compounds in Scots pine (Pinus sylvestris L.). Scientific Reports 8, (2018). 